\title{
CORRIGENDUM: Synthetic fluorescent sensors for studying the cell biology
} of metals

Dylan W Domaille, Emily L Que \& Christopher J Chang

Nat. Chem. Biol. 4, 168-175 (2008); published online 15 February 2008; corrected after print 26 June 2008

In the version of this article initially published, the chemical structures for S3 and RS2 in Figure 6 were incorrect. The error has been corrected in the HTML and PDF versions of the article. 